\title{
Comparison of estimation capabilities of the artificial neural network with the wavelet neural network in lipase-catalyzed synthesis of triethanolamine-based esterquats cationic surfactant
}

\begin{abstract}
In this study, estimation capabilities of the artificial neural network (ANN) and the wavelet neural network (WNN) based on genetic algorithm were investigated in a synthesis process. An enzymatic reaction catalyzed by Novozym 435 was selected as the model synthesis process. The conversion of enzymatic reaction was investigated as a response of five independent variables; enzyme amount, reaction time, reaction temperature, substrates molar ratio and agitation speed in conjunction with an experimental design. After training of the artificial neurons in ANN and WNN, using the data of 30 experimental points, the products were used for estimation of the response of the 18 experimental points. Estimated responses were compared with the experimentally determined responses and prediction capabilities of ANN and WNN were determined. Performance assessment indicated that the WNN model possessed superior predictive ability than the ANN model, since a very close agreement between the experimental and the predicted values was obtained.
\end{abstract}

Keyword: Artificial neural network (ANN); Cationic surfactant; Esterquats; Estimation capabilities; Optimization; Wavelet neural network (WNN) 